PublMed.gov

US National Library of Medicine National Institutes of Health
PubMed

Diversity in the Scope and Practice of Hospital-Based Midwives in the Netherlands

Search
Display Settings: $\nabla$ Abstract

J Midwifery Womens Health. 2012 Sep-Oct;57(5):469-75. doi: 10.1111/j.1542-2011.2012.00164.x.

\section{Diversity in the scope and practice of hospital-based midwives in the Netherlands.}

$\underline{\text { Cronie D, }}$ Rijnders M, Buitendijk S.

doug.cronie@tno.nl

\section{Abstract}

INTRODUCTION: Not all midwives in the Netherlands are independent practitioners. One in 4 midwives registered to practice is employed in the hospital setting, where $67 \%$ of all births occur. There has not yet been an in-depth examination of hospital-based midwives' practice in the Netherlands, in the context of care in a higher-risk environment. The primary aims of this study were to describe the diversity and scope of practice of hospital-based midwives in the Netherlands.

METHODS: This was an online survey of all hospitals throughout the Netherlands with labor/birthing rooms and employing hospital-based midwives. The survey covered 5 topic areas: demographic/organizational details, duties, responsibilities, experience/additional qualifications, and how the midwife functioned within the multidisciplinary hospital team. Descriptive statistics are provided.

RESULTS: A total of 59 secondary and tertiary level hospitals from a possible total of 98 were included for analysis (60\% national response rate). Forty percent of all births occurring during the study period were managed solely by a hospital midwife. The provision of midwifery care in the hospital setting was not universal, and where present, hospital-based midwives were not necessarily available 24 hours a day or 7 days a week. Hospital-based midwives reported a high level of autonomy.

DISCUSSION: Currently there is no universal provision of midwifery care in the hospital setting in the Netherlands. Where there are hospital-based midwives, they appear to manage the majority of births. However, there are no nationally agreed-upon standards for midwifery practice in the hospital setting, and no agreement exists over minimum requirements relating to additional education for midwives in these settings. A national evaluation and setting of minimum standards is needed.

(C) 2012 by the American College of Nurse-Midwives.

PMID: 22954077 [PubMed - indexed for MEDLINE]

Publication Types, MeSH Terms

LinkOut - more resources

\section{Full Text 9WILEY If Online}

Leiden $\mathrm{S}$ S.F.X
Add to Favorites

Related citations in

PubMed

Evaluation of primary care mi [BMC Health Serv Res. 2012]

An evaluation of the satisfaction of midwives' wc [Midwifery. 2010]

The development of national competency st: [Midwifery. 2007]

Review Routine examination of tr [Health Technol Assess. 2004]

Review Intrapartum care in the twen [Nurs Clin North Am. 2002]

See reviews...

See all..

\section{Search details}

Diversity[Title] AND Scope[Title] AND Practice[Title] AND Hospital-Based[Title] AND Midwives[Title] AND Netherlands[Title]

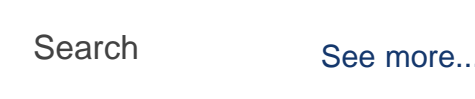

Recent activity

$\underline{\text { Turn Off } \text { Clear }}$

Diversity[Title] AND Scope[Title] AND PubMed

Q Diversity in the Scope and Practice of Hospital-E PubMed

Diversity in the scope and practice of hospital-bi PubMed 
You are here: NCBI > Literature > PubMed
GETTING STARTED

NCBI Education

NCBI Help Manual

NCBI Handbook

Training \& Tutorials

\section{RESOURCES}

Chemicals \& Bioassays

Data \& Software

DNA \& RNA

Domains \& Structures

Genes \& Expression

Genetics \& Medicine

Genomes \& Maps

Homology

Literature

Proteins

Sequence Analysis

Taxonomy

Training \& Tutorials

Variation

\section{POPULAR}

PubMed

Nucleotide

BLAST

PubMed Central

Gene

Bookshelf

Protein

OMIM

Genome

SNP

Structure
FEATURED

Genetic Testing Registry

PubMed Health

GenBank

Reference Sequences

Map Viewer

Human Genome

Mouse Genome

Influenza Virus

Primer-BLAST

Sequence Read Archive
Write to the Help Desk

NCBI INFORMATION

About NCBI

Research at NCBI

NCBI Newsletter

NCBI FTP Site

$\mathrm{NCBI}$ on Facebook

NCBI on Twitter

NCBI on YouTube

\section{Copyright | Disclaimer | Privacy | Browsers | Accessibility | Contact}

National Center for Biotechnology Information, U.S. National Library of Medicine 8600 Rockville Pike, Bethesda MD, 20894 USA

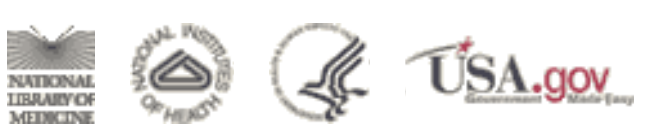

